\title{
A CFD and Experimental Study of Thin Flexible Wire in a Cross Flow
}

\author{
Chelakara S. Subramanian*, Harika Gurram, Priyanka L. Kanherkar \\ Florida Institute of Technology \\ MAE Department, $150 \mathrm{~W}$. University Boulevard, Melbourne, FL, USA \\ subraman@fit.edu; hgurram2015@my.fit.edu; pkanherkar2014@my.fit.edu.
}

\begin{abstract}
This research focuses on understanding the drag of thin flexible wires in a cross flow at low laminar Reynolds numbers. Experiments are conducted to measure the drag force for thin flexible wire of $\mathrm{O}(\mathrm{mm})$ at Reynolds number, based on wire diameter, range between 250 to 1000 and, the results showed $20 \%$ to $30 \%$ of reduction in the drag coefficient as compared to previous quoted results in the open literature. The direct numerical simulation study is carried out for the similar flow (but for a less flexible wire) using the commercial CFD code ANSYS Fluent 15.0 and with mesh generation ANSYS ICEM-CFD 15.0 software. The computational results confirmed the experimental results. To further explain the fluid dynamic mechanism causing the drag reduction in this range of Reynolds numbers, wake surveys are performed using pitot static tube and hot wire experiments as well as time resolved computational numerical simulations. The near wake flow structure suggests the wake transition may be the cause of reduced drag, similar to the drag crisis commonly observed in the wire boundary layer transition regime.
\end{abstract}

Keywords: Drag coefficient; Flexible cylinder drag; CFD; Low Reynolds number wake transition; Hot wire wake survey

\section{Introduction}

Thin flexible wires are used in applications such as underwater fiber-optic cables, electrical transmission lines, mooring buoys and tethering objects, and in convective heating and cooling systems. Many studies, e.g. [1], have been performed for rigid wires or thin cylinders but measurements on flexible cylinders are not available. Measurements of drag coefficients of thin flexible cylindrical wires are described in [2] for the Reynolds number range between $250-1000$. Their results indicate that the coefficient values are about 20 to 30 percent lower than the reported laminar flow values for rigid cylinders. Previous researches on low-Reynolds number cylindrical cross flows suggest the drag coefficient is largely influenced by the boundary layer instability. A drag crisis occurs when the boundary layer on the cylinder transits at critical Reynolds number. The common understanding of this flow is that at subcritical Reynolds numbers the laminar boundary layer separation occurs earlier than at the supercritical Reynolds numbers, resulting in reduced wake width and pressure drag. This appears as the drag crisis in the Cd versus Re plot. The present authors hypothesize that a similar but less severe drag reduction occurs due to wake flow transition at very low Reynolds numbers. The notable related previous studies are by Bengt Forenberg [3], who used a numerical approach to compute the drag coefficient for a steady viscous flow past a circular cylinder with small diameters and observed low drag coefficient values for 100 - 300 Reynolds numbers (Figure 1). Williamson [4] observed a dip in the base pressure coefficient of a 2D cylinder (see the dip between $\mathrm{B}-\mathrm{C}$ in Figure 2) for similar range of Reynolds numbers. He attributes this to the wake unsteadiness. Experiment conducted by Finn [5] in 1953 also discusses the drag on cylinder at low Reynolds numbers. To measure the drag on the cylinder they have used a fine wire suspended as a pendulum in a uniform stream of air flowing at a known rate. The drag force is measured from the amount of deflection. All these results enlightens the importance of understanding the wake structure of small diameter cylinder at low Reynolds numbers. Previous work reported by researches are mainly for the rigid cylinder with comparatively larger diameters. The present work's emphasis is on acquiring an explicit view for the formation of wake structure behind thin flexible wire. The details of mean, root-mean squared (RMS) velocities, vorticity and separation locations causing the drag reduction are described here based on the observed results of CFD simulations of flow on rigid and flexible thin cylinders and experimental pitot-static tube and hotwire wake surveys. 


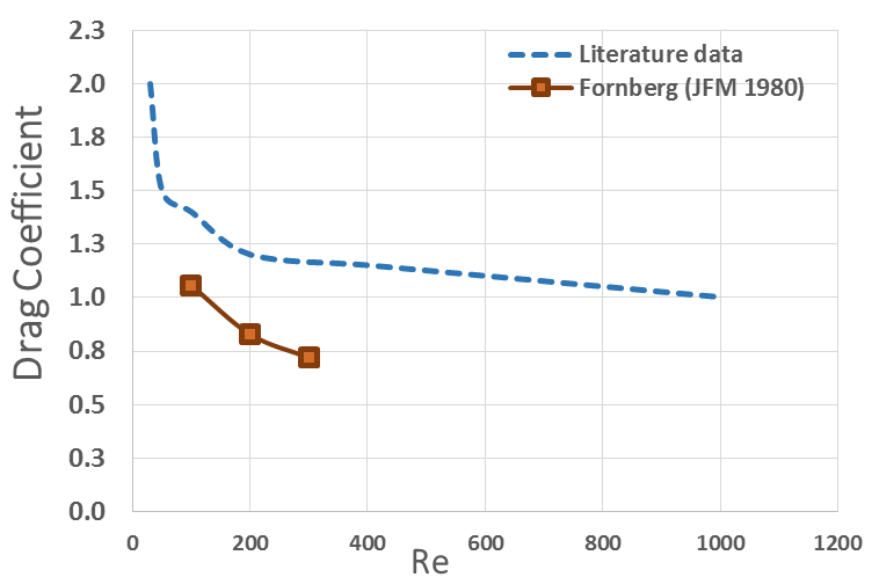

Fig. 1: Bengt Forenberg [3] data.

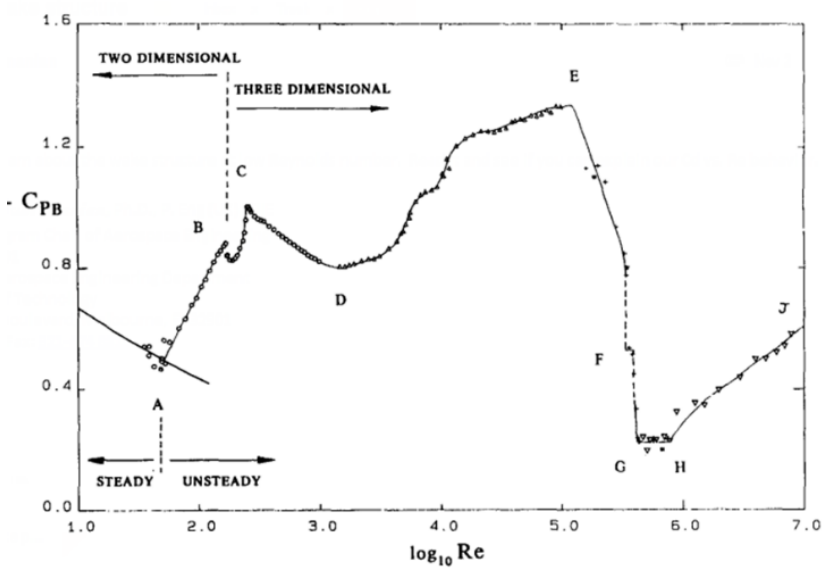

Fig. 2: Williamson [4] data.

\section{Experimental Description}

The experiments are conducted in the Florida Tech low subsonic wind tunnel facility (Figure 3a) with a test section of cross section $0.54 \mathrm{~m} \times 0.54 \mathrm{~m}$ and $1.72 \mathrm{~m}$ length. Four flexible cables with the diameters 750, 800, 1000 and 1150 microns, respectively, are stretched taut across the test section. The freestream speed for the wake survey tests is varied between $5 \mathrm{~m} / \mathrm{s}$ to $18 \mathrm{~m} / \mathrm{s}$. This yielded the Reynolds number based on the freestream speed and wire diameter variation from 210 to 1156 as shown in Table 1. For the drag determination by the momentum balance, the dynamic pressure (hence the mean velocity) in the wire wake are measured by traversing a $3 \mathrm{~mm}$ tip diameter semi-hemispherical pitot static tube (Figure 3b) and using a pressure transducer. The pitot static tube wake survey is carried out with a manual traverser, using $0.1 \mathrm{~mm}$ step size in the wake region. For the averages, a hundred samples at sampling rate of 5 samples per second are used. The wake surveys are performed for $5 \mathrm{~mm}, 8 \mathrm{~mm}$ and, $10 \mathrm{~mm}$ stream wise locations. For the turbulent velocity measurements in the wake, a single $5 \mu \mathrm{m}$ Platinum plated tungsten straight wire operated by a constant temperature anemometer is used (shown in Figure 3c). The wire is calibrated in the potential flow region of the wind tunnel for speed range from $4 \mathrm{~m} / \mathrm{s}$ to $18 \mathrm{~m} / \mathrm{s}$. A linearized calibration constants is used to reduce the measured voltage data to velocity. For the hot wire wake survey an automated traverse with $0.1 \mathrm{~mm}$ step size is used inside the wake. Twenty thousand samples of instantaneous velocities are collected at 10,000 samples per second of Nyquist frequency. This assumes a useful resolved frequency of about $5 \mathrm{kHz}$. The National Instruments data acquisition system (CompactDAQ) with two different LabView programs (one for the pitot-static tube survey and one for hotwire survey) are used for the data processing. Figure 4 is a screen shot of the mean and RMS velocity from a hotwire run.

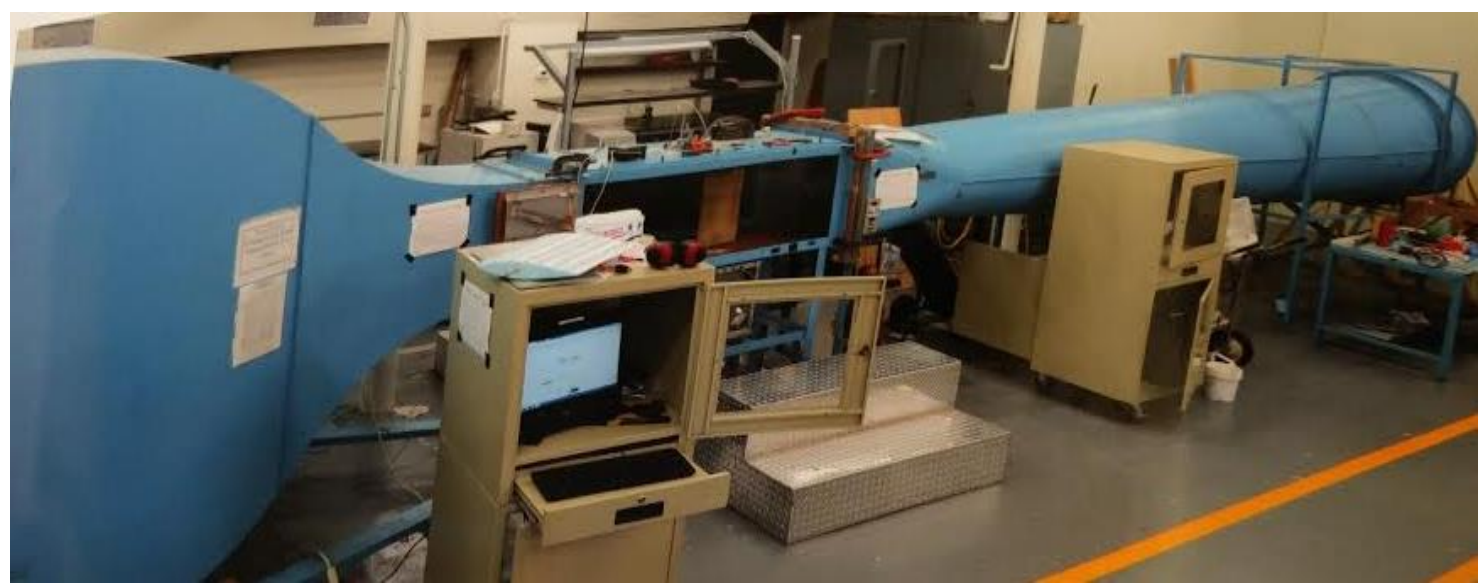

Fig. 3(a): Low subsonic wind tunnel. 


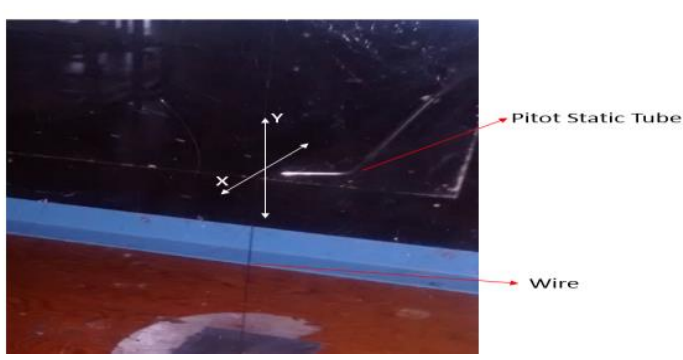

Fig. 4(b): Pitot tube setup (note $\mathrm{X}$ axis is across the wake).

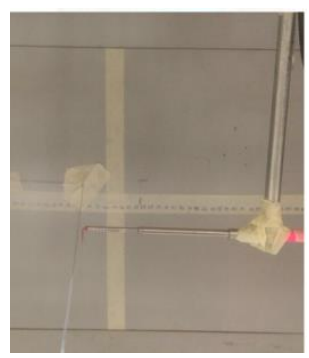

Fig. 5(c): Hot wire setup (note $\mathrm{Y}$ axis is across the wake).

Fig. 6: The Experimental Setup.

Table 1: Experimental test variables.

\begin{tabular}{|c|c|c|}
\hline Diameter, $\mu \mathrm{m}$ & Velocity, $\mathrm{m} / \mathrm{s}$ & $\mathbf{R e}$ \\
\hline 750 & 5 & 209.5 \\
\hline 800 & 5 & 223.5 \\
\hline 1000 & 5 & 279.3 \\
\hline 1150 & 5 & 321.2 \\
\hline 750 & 7.5 & 314.2 \\
\hline 800 & 7.5 & 335.2 \\
\hline 1000 & 7.5 & 419.0 \\
\hline 1150 & 7.5 & 481.8 \\
\hline 750 & 10 & 419.0 \\
\hline 800 & 10 & 446.9 \\
\hline 1000 & 10 & 558.7 \\
\hline 1150 & 10 & 642.5 \\
\hline 750 & 13.5 & 565.6 \\
\hline 800 & 13.5 & 603.4 \\
\hline 1000 & 13.5 & 754.2 \\
\hline 1150 & 13.5 & 867.3 \\
\hline 750 & 15 & 628.5 \\
\hline 800 & 15 & 670.4 \\
\hline 1000 & 15 & 838.0 \\
\hline 1150 & 15 & 963.7 \\
\hline 750 & 18 & 754.2 \\
\hline 800 & 18 & 804.5 \\
\hline 1000 & 18 & 1005.6 \\
\hline 1150 & 18 & 1156.4 \\
\hline
\end{tabular}

\section{Computational Studies}

A systematic computational study is done for above mentioned diameters and velocity range to investigate the wake structure and its effect on the drag coefficient. The Direct Numerical Simulation (DNS), pressure based steady state solver with second order upwind flow discretization is used to compute all the test cases and the results are compared to the experimental results. The analysis is done considering a stiff cylinder and a flexible cylinder to account for the elastic deformation. The details of the simulations and results are briefed out in the following sections.

\subsection{Grid Independence}

The gird Independence study was conducted to validate the case setup. The theoretical curve for $25 \mathrm{~mm}$ diameter cylinder is used and simulated for the Reynolds number range from 50 to 1000. For this, a total of four different 2Dstructured grids of the following sizes were used, grid1 ( 10000 elements), grid2 ( $\sim 50000$ elements), grid3 ( 150000 
elements), grid4 ( 450000 elements). The Figure 5a shows the comparison of the results of Cd vs Re for the above given grid sizes with respect to the theoretical data. The simulation with grid 3 gave the most approximate results, hence this grid size and setup is used to calculate the drag coefficients for all the other diameters.

\subsection{Stiff Cylinder Simulation}

The simulations to observe the effect of the wake structure on the Reynolds number are performed by varying the diameter and velocities for 2-D cylinder (diameters 750, 800, 1000 and 1150 microns) for velocity range $5 \mathrm{~m} / \mathrm{s}$ to $18 \mathrm{~m} / \mathrm{s}$. From these simulation the dynamic pressure and velocity variation were extracted at downstream locations of $5 \mathrm{~mm}, 8 \mathrm{~mm}$ and $10 \mathrm{~mm}$. The computational results in Figure 5b show a similar drag reduction compared to the experiment.
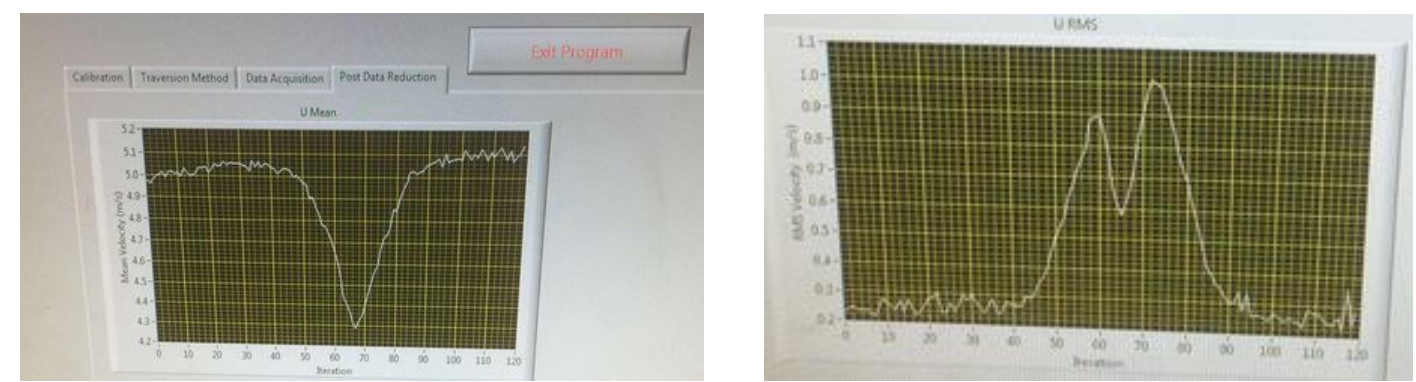

Fig. 7: Screen plot of Mean (left) and RMS (right) velocities from program output of the hot wire run.

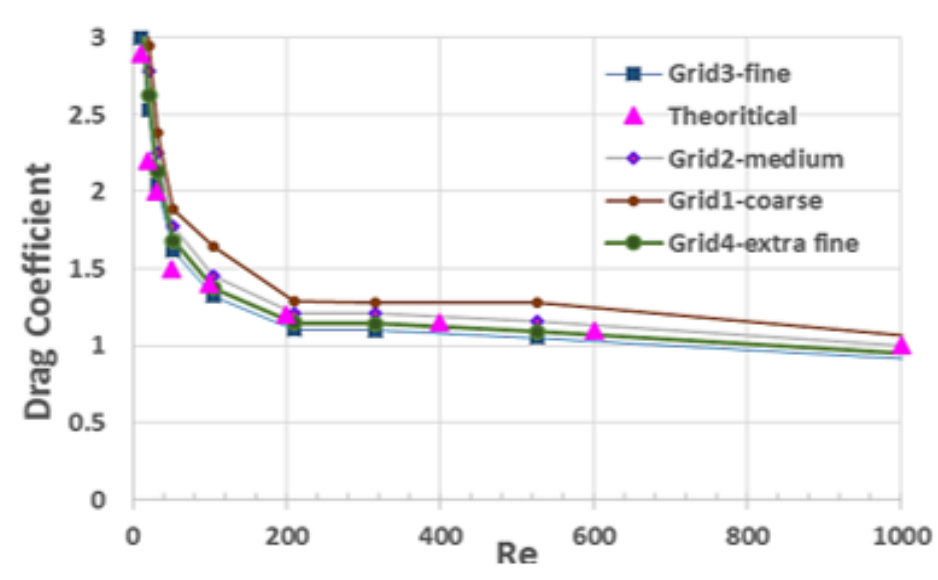

Fig. 8(a): Grid independent study results.

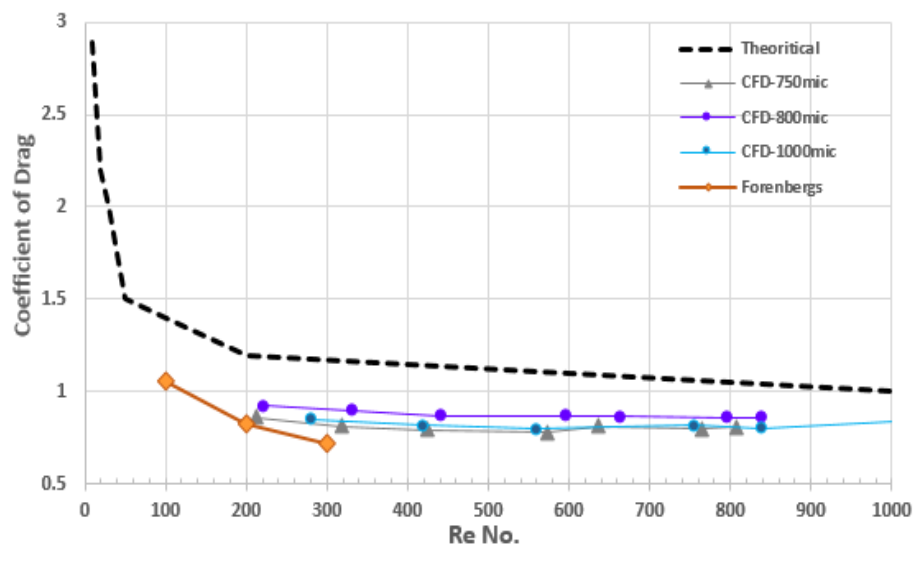

Fig. 9(b): Coefficient of drag vs Re for different diameters.

\subsection{Fluid Structure Interaction}

The fluid structure interaction (FSI) simulations for different wire stiffness are conducted on a 3-D cylinder to observe for the effect of wire deformation on wake flow. To do this, a one-way FSI case setup is used. This case setup starts with fluent (DNS) simulation which solves for the pressure loads on the cylinder at a given velocity, then the solution and geometry details from fluent are transported to the static structural module which takes the pressure loads as an input and solves for the deformation of the cylinder. The total deformation value depends upon the given material Young's modulus. The deformation value is then fed into the mechanical APDL analysis which gives the deformed node values. This is further directed to finite element modeller (FE modeller) which models the deformed structure by deleting the mesh. Finally, this geometry is transformed back to the fluent file to update the drag values for the maximum deformed structure. Figure 6 shows the sample wire deformation for different velocity. 


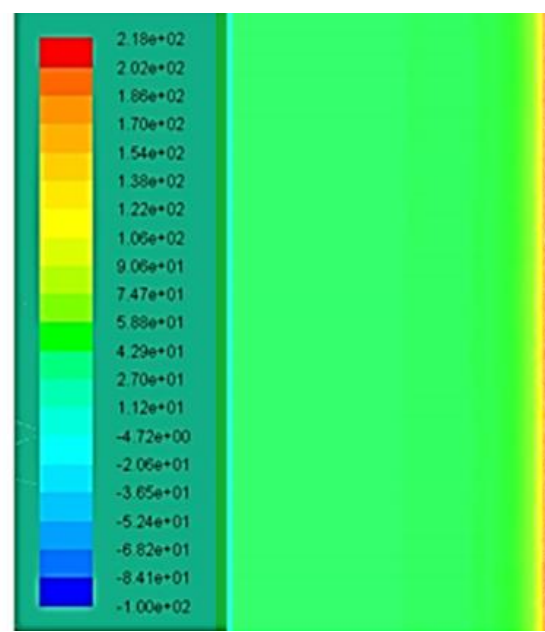

(a)

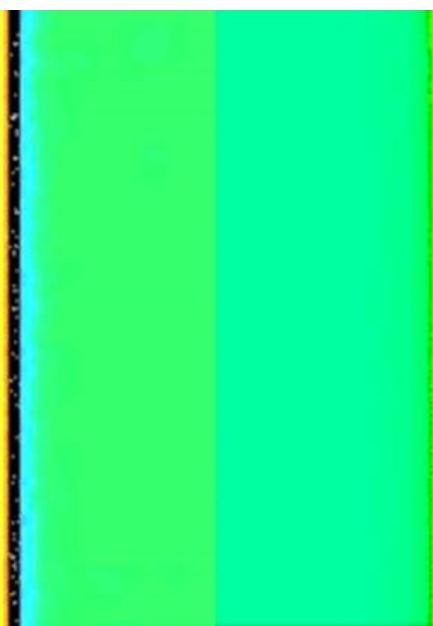

(b)

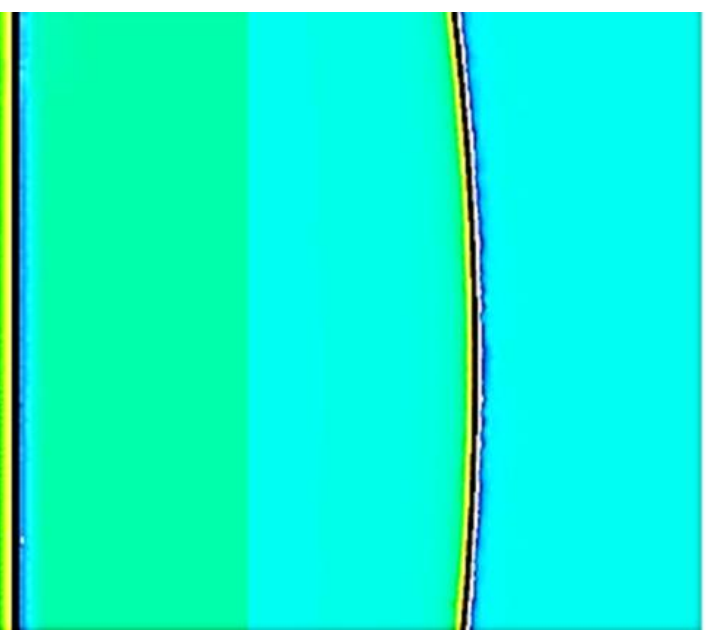

(c)

Fig. 6: Static pressure distribution around the wire (dia $=1000$ micros) before and after deformation.

(a) Shows the un-deformed wire (b) shows the minimum deformation of $0.08 \mathrm{~mm}$ at $\mathrm{v}=5 \mathrm{~m} / \mathrm{s}$ and (c) shows the maximum deformation of $0.5 \mathrm{~mm}$ at $\mathrm{v}=18 \mathrm{~m} / \mathrm{s}$.

\section{Results and Discussions}

The Reynolds number variation can be achieved by either changing the velocity or by changing the diameter or by altering both velocity and diameter. In the present case we change the Reynolds number by varying both velocity and diameter and obtained the drag coefficient results for Reynolds number ranging from 50 to 1000. Figure 7a shows the plot of drag coefficient versus Re and Figure $7 \mathrm{~b}$ shows plots of dynamic pressure (from pitot static tube) ratio distribution in the wake of 800 micron cylinder for various freestream speeds. It is observed that increasing the Reynolds number by increasing velocity causes very little change in the wake width. However the maximum velocity deficit increases with the Reynolds number. Figures 8-13 show the plots of hotwire measurements for different velocities and stream wise locations. There appears to be no similarity at $5 \mathrm{~mm}$ location for both mean and intensity profiles. The width of the wake hardly changes with the distance. It is noticed that the maximum RMS velocity occurs away from the wake axis. Figure 14 shows the CFD simulation results wake of development with distance and velocity. As in the experiments here also the velocity deficit increase as the flow moves towards the downstream. At higher speeds the mean velocity profile attains a Gaussian shape for all distances. To investigate the effect of Reynolds number change due to diameter change a CFD analysis is performed for varying diameters 100 to 1000 microns. In the course of this study it is observed that the drag coefficient value is affected by the wake structure and the boundary layer occurring on the wire. In Figure 15a, the flow separation angle is determined by considering the zero wall shear stress in the wire boundary layer for all the diameters. The Figure $15 \mathrm{~b}$ shows the plot for separation angle $\left(\theta^{\circ}\right.$, measured from front stagnation) vs the Reynolds number for different diameters. For all the cases the separation angle is greater than $90^{\circ}$ which suggests that the wake flow unsteadiness may be altering the base pressure and delaying separation around the cylinder. However, as the Reynolds number increases (for the range considered here) the separation angle decreases, thereby increasing the wake width. This observation is consistent with Williamson's [4] results which show that there is a slight dip in the base pressure due to unsteady wake beyond Reynolds number of about 200. These observations and results encourage to further investigate the nature of the wake and its interaction with the boundary layers. The Figure 16 shows the velocity contours from the CFD simulations for 3 diameters and 3 different velocities i.e. $5 \mathrm{~m} / \mathrm{s}, 10 \mathrm{~m} / \mathrm{s}$ and $18 \mathrm{~m} / \mathrm{s}$, at low velocity and small diameter the vortex shedding can be observed but at higher velocity and large diameter the vortex is not clearly formed. 


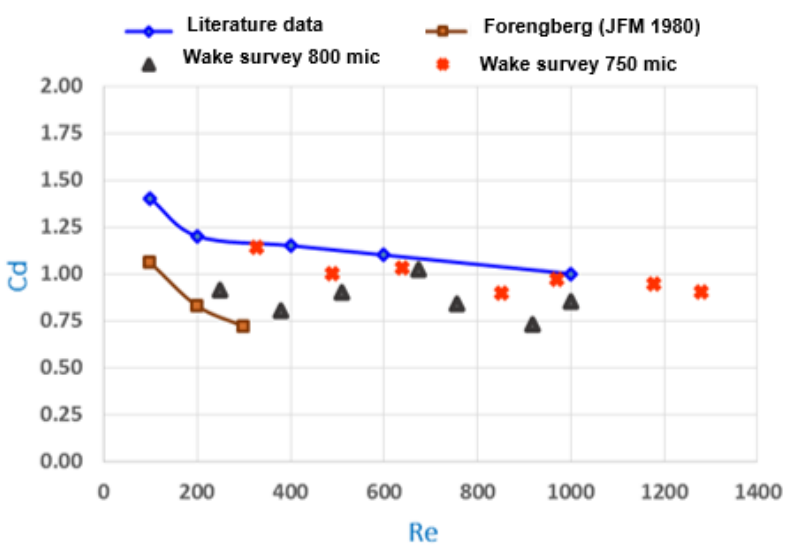

(a)

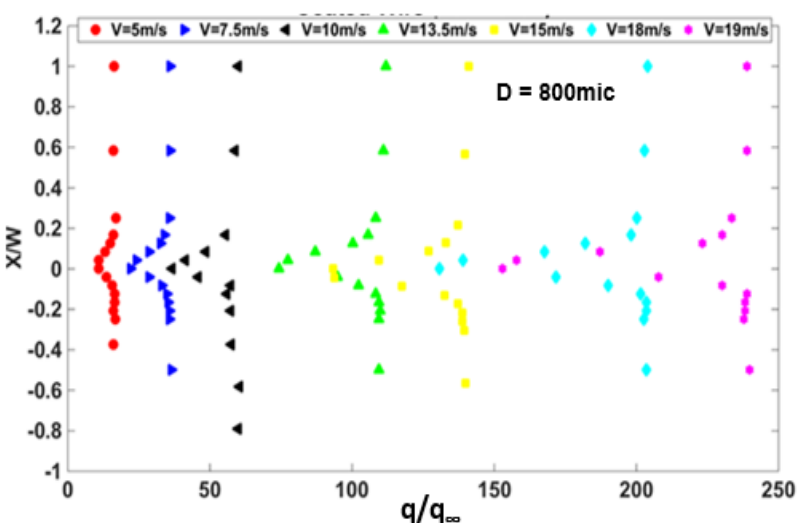

(b)

Fig. 7: Wake survey results (a) Coefficient of drag vs Re no for different diameters, (b) Dynamic pressure variation in the wake region for wire of diameter of 800 microns.

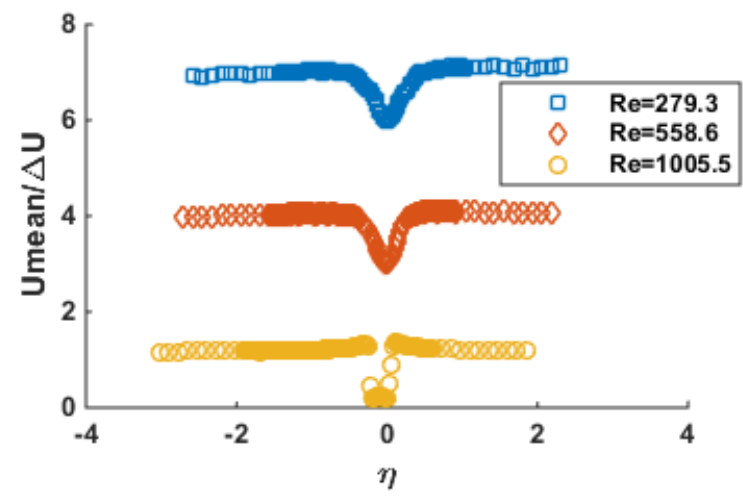

Fig. 8: Mean velocity variation vs eta @ 5mm.

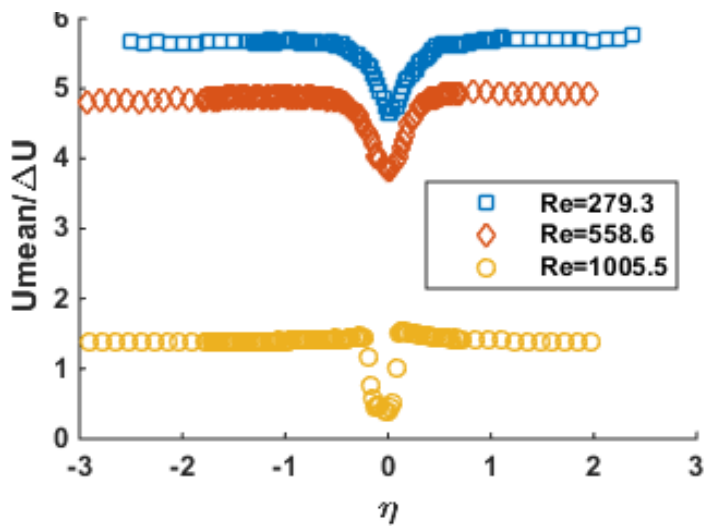

Fig. 10: Mean velocity variation vs eta @ $8 \mathrm{~mm}$.

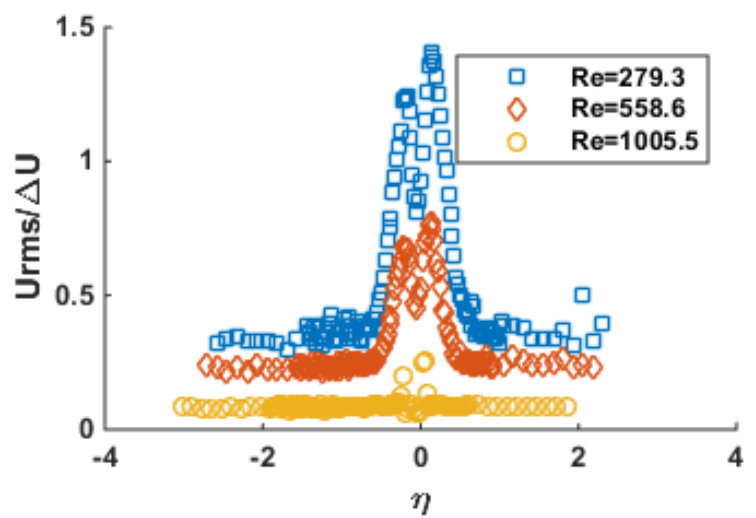

Fig. 9: Turbulence intensity variation vs eta @ 5mm.

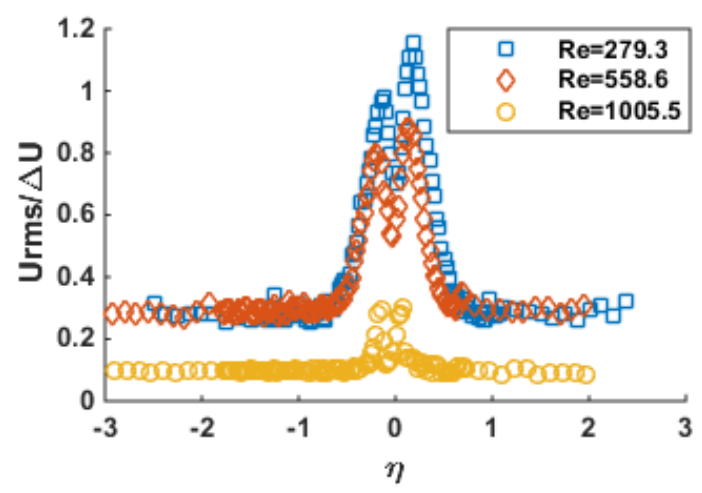

Fig. 11: Turbulence intensity variation vs eta @ 8mm. 


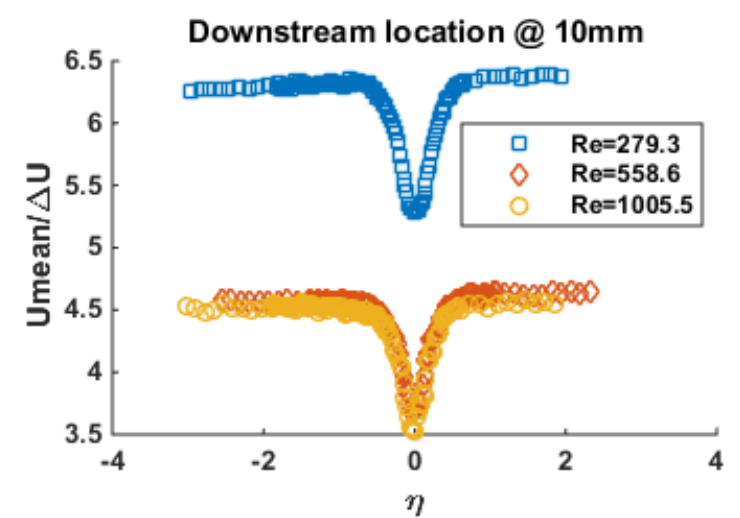

Fig. 12: Mean velocity variation vs eta @ $10 \mathrm{~mm}$.

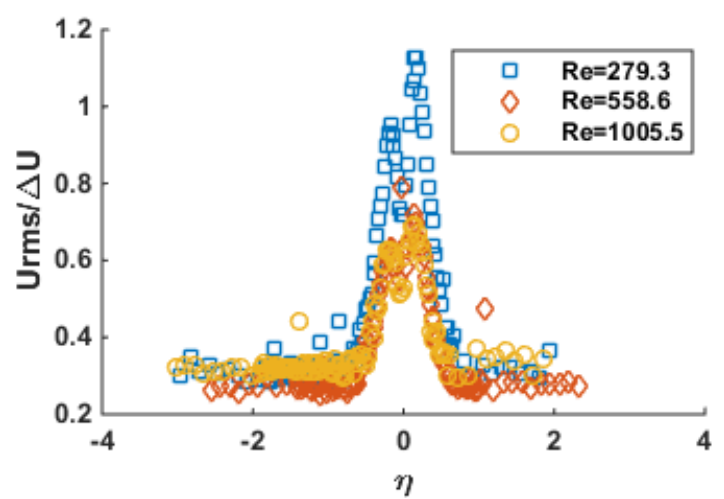

Fig. 13: Turbulence intensity variation vs eta @ 10mm.
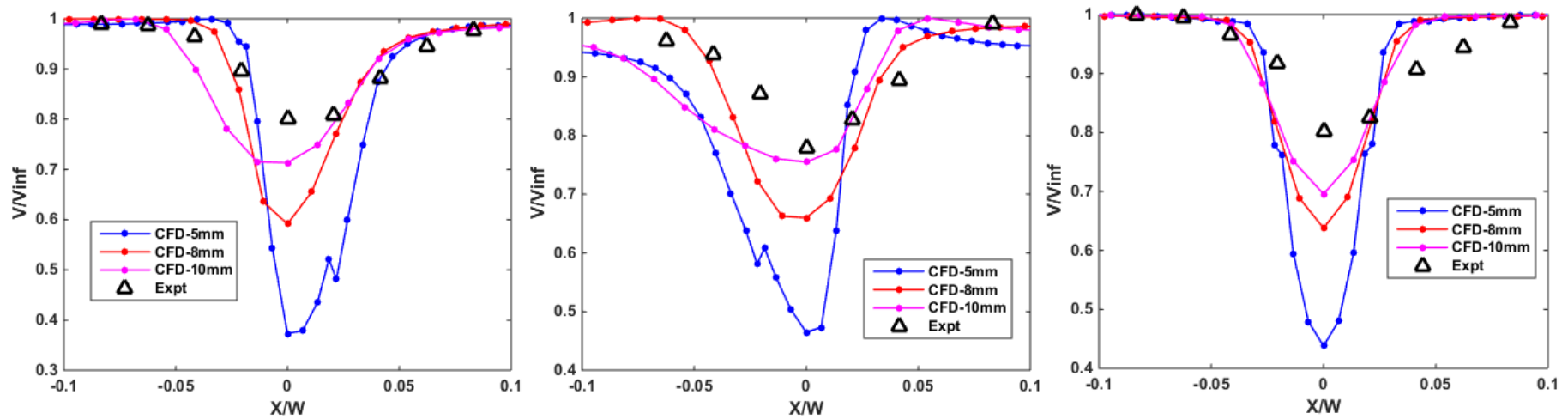

Fig. 14: CFD and experimental comparison of wake at different downstream locations for $\mathrm{v}=5 \mathrm{~m} / \mathrm{s}$ (left), $10 \mathrm{~m} / \mathrm{s}$ (center), and $18 \mathrm{~m} / \mathrm{s}$ (right) respectively.
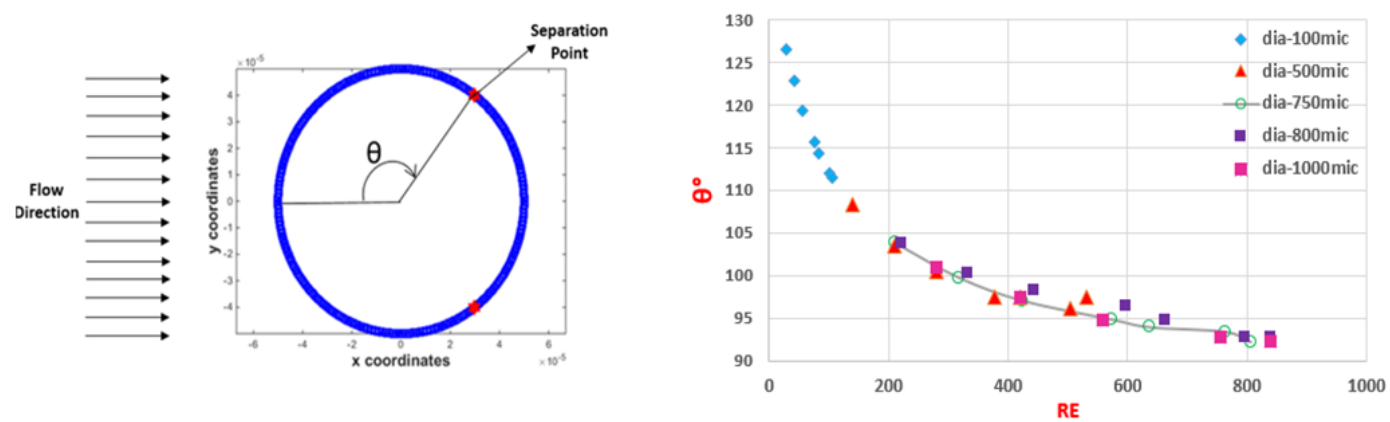

Fig. 15(a): The flow separation angle measurement. Fig. 15(b): The flow separation angle versus Re.

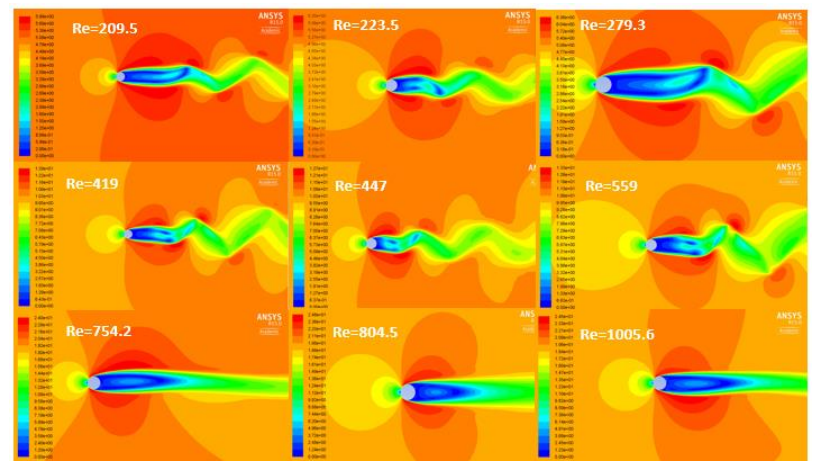

Fig. 16: Velocity magnitude contour plots. 


\section{Conclusions}

The drag coefficient of thin flexible cylindrical wire in a cross flow is investigated for Reynolds numbers 200 to 1000. Experiments using pitot static tube and single hotwire anemometer and, the DNS simulations are used for the study. Compared to the classical results, about 20 to 30 percent reduction in the drag coefficient is observed at Reynolds numbers between 150 and 300. The evidence of unsteady wake flow structure leading to turbulence in the wake nearfield is believed to be responsible for the local drag reduction. There seems to be very little wake growth with velocity in the nearfield. Semblances of periodic narrowband unsteadiness (vortex shedding) is noticed at lower Reynolds numbers. As the Reynolds number increases, the periodic unsteadiness changes to a broadband random unsteadiness, leading to a fully turbulent wake.

The fluid structure interaction study gave small deflection values and no significant effect on the drag coefficient. But the variation in wake structure due to the deflection should be further investigated.

\section{References}

[1] A. Roshko, "Experiments on the flow past a circular cylinder at very high Reynolds number," Journal of Fluid Mechanics, vol. 10, no. 3, pp 345-356, 1961.

[2] C. Subramanian and H. Gurram, "Drag Coefficient of Thin Flexible Cylinder," in Proceedings of the 68th Annual Meeting of the APS Division of Fluid Dynamics, Hynes Convention Canter, Boston, MA, 2015, paper no. DFD152015-000929, vol. 60, no. 21.

[3] B. Fornberg, "Numerical study of steady viscous flow past a circular cylinder," J Fluid Mech, vol. 98, no. 4, pp 819$855,1980$.

[4] C. H. K. Williamson, "Vortex Dynamics in the cylinder wake," Ann. Re. Fluid Mech, vol. 28, pp. 477-539, 1996.

[5] R. K. Finn, "Determination of the drag on a cylinder at low Reynolds no.," Jour. of applied physics, vol. 24, no. 6, 1953. 